\title{
Use of Pregabalin as a Pre-Medication for Post-Operative pain in patients undergoing Laparoscopic Cholecystectomy
}

\author{
Tabish Hussain ${ }^{1}$, Asifa Anwar Mir ${ }^{2}$, Jawad Zahir ${ }^{3}$, Pervaiz Minhas ${ }^{4}$ \\ ${ }_{1}^{1}$ Head, Department of Anesthesia, ICU and Pain Medicine, Fauji Foundation Hospital, Kallar Kahar \\ ${ }^{2}$ Registrar, Department of Anesthesia, ICU and Pain Medicine, Fauji Foundation Hospital, Kallar Kahar \\ ${ }^{3} \mathrm{Head}$, Department of Anesthesia, Intensive Care and Pain Medicine, Rawalpindi Medical College. \\ ${ }^{4}$ Anesthetist, Department of Anesthesia, Intensive Care and Pain Medicine, Rawalpindi Medical College
}

\begin{abstract}
Background: Postoperative pain creates complications by increasing circulating level of catecholamines and systemic vascular resistance, thus putting the patients on increased risks of having stroke and myocardial infarction. In addition, it increases hospital stay, causing burden over economic as well as healthcare infrastructure. The aim of this study was to determine the frequency of pain in the postoperative period while using Pregabalin as pre-medication among patients undergoing laparoscopic cholecystectomy.

Material and Methods: The randomized control trail was conducted at Department of Anesthesiology, Holy Family hospital, Rawalpindi from $1^{\text {st }}$ Sept 2015 to $28^{\text {th }}$ Feb 2016 over a period of 6 months. A total of 200 patients undergoing laparoscopic cholecystectomy were randomly divided in group A and B by consecutive non-probability lottery method. Group A received $100 \mathrm{mg}$ oral Pregabalin 1 hour before surgery and Group B were not given Pregabalin and were taken as controls. Post-operative pain was measured by visual analog scale (VAS) in terms of pain scores at 4 hours postoperatively after the arrival of patient in the postanesthesia care unit (PACU). SPSS version 17.0 was used to analyze the data.

Results: A total of 200 patients were included in the study. There were 100 patients in each group. Based on the visual analog pain scores, 9 patients were pain free in group A compared with none in group B. Similarly, there were 55 patients in group A, who reported a pain score of 1 whereas no patient in group B had a VAS score of 1 . There were 29 patients in group A and only 3 patients in group $B$ with VAS score of $2(90.6 \%$ vs. $9.4 \%)$. For VAS score of 3 , there were 6 patients in group $A$ and 34 patients from group B (15\% vs. $85 \%)$. For a VAS score of 4 , there were 1 patient in group A and 61 patients in group B (1.6\% vs. 98.4\%). Two patients in group $B$ experienced a VAS score of 5 . All this data was significant with chi square $p$ value of 0.0001 .

Conclusion: Oral Pregabalin administered prior to laparoscopic cholecystectomy was effective in reducing postoperative pain in the patients. Further studies are needed for post-operative evaluation of side effects, different dosing schedules at different time intervals for both rest and dynamic pain.
\end{abstract}

Key words: Laparoscopic Cholecystectomy; Pregabalin; Pre-Medication; Post-operative pain.

Authors' Contribution:

1,2 Conception, synthesis, planning of

research and manuscript writing ${ }^{3,4}$ Interpretation, discussion, Data analysis, Active participation in data collection.

Cite this article. Hussain T, Mir AA, Zahir J, Minhas P. Use of Pregabalin as a Pre-Medication for Post-Operative pain in patients undergoing Laparoscopic Cholecystectomy. J Islamabad Med Dental Coll.2019; 8(1):29-33

\section{Introduction}

Pain is one of the commonest post-operative complaints all around the world after major surgical procedures. ${ }^{1}$ The post-operative pain control has a direct relationship with postoperative morbidity, reflecting its importance regarding time and adequate management. ${ }^{2}$ Postoperative pain is involved in creating complications
Article info:

Received: November 11, 2018

Accepted: February 10, 2019

Funding Source: Nil

Conflict of Interest: Nil 
by increasing circulating level of catecholamines and systemic vascular resistance, thus putting the patients at increased risks of having stroke and myocardial infarction. ${ }^{3}$ In addition, it increases hospital stay, which in turn cause burden over economic as well as healthcare infrastructure. The control of pain has been given less priority as reflected by number of health care programs conducted for pain education and its funding resources. ${ }^{4}$ Early post-operative pain can decrease early ambulation, slows bowel function and be a major problem in early discharge of patients. ${ }^{5}$ Inadequate post-operative pain control may also lead to the development of chronic pain after surgery. Thus, attenuation of early post-operative pain remains an important concern for medical personal especially the anaesthtetist. ${ }^{5}$

Pain after laparoscopic surgeries, especially laparoscopic cholecystectomy is a common complaint. ${ }^{6}$ Various analgesics have already been tried for the control of postoperative pain among these patients ranging from simple Non-Steroidal Anti-Inflammatory Drugs (NSAIDs) to strong opioids, but none of them is specific and the adequate control of such pain is only possible with multimodal approach. Some recent studies have shown that use of Pregabalin as premedication can effectively control the postoperative pain and consumption of rescue analgesic medications after surgical procedure, and among patients undergoing general anesthesia for laparoscopic cholecystectomy. . $^{7,8}$

A variety of post-operative anesthetic strategies ranging from simple analgesic agents administered orally to block nerve for pain management have been sought. ${ }^{9} \mathrm{~A}$ simpler strategy of preemptive analgesic agent may be more acceptable for both anesthetist and patients as a reliable pain relief technique. ${ }^{10}$ Preemptive analgesia is defined as an anti-nociceptive treatment that prevents establishment of altered central processing of afferent input from injuries. ${ }^{11}$ This can effectively prevent earlier onset of the pain than that of the preventive administration of the drugs after surgical procedures, but it was controversial whether preemptive or preventive analgesia should be used to describe the difference between the two analgesic techniques. ${ }^{12,13,14}$

Gabapentene and its derivate Pregabalin had successfully been used in control of neuropathic pain in the past. Pregabalin is a GABAergic drug that acts on the GABA receptors at the level of spinal cord and brain to inhibit the pain signals. ${ }^{15}$ They bind to the pre-synaptic voltage gated calcium channels, thus causing reduction of calcium influx leading to reduction in release of various excitatory neurotransmitters. It has rapid extensive absorption after oral intake, $90 \%$ bioavailability with maximal plasma levels achieved after about 1 hour. The efficacy of Pregabalin has only been observed for limited procedures as in control of postoperative pain after laparoscopic hysterectomy orthopedic spinal fusion surgery and the dental procedures. ${ }^{16,17}$ The role of Pregabalin for Post-operative cholecystectomy has not yet been studied in detail especially in Pakistan, where cholelithiasis is an emerging issue because of change in life style and dietary habits. The purpose of this study was to evaluate the effectiveness of pre-operative analgesia using Pregabalin by measuring the frequency of postoperative pain following laparoscopic cholecystectomy.

\section{Material and Methods}

This randomized control trial was conducted at Department of Anesthesiology, Holy Family hospital, Rawalpindi from $1^{\text {st }}$ September 2015 to $28^{\text {th }}$ February 2016 over a period of 6 months. Approval from Institutional Ethics Committee was taken at the start of this study. After written informed consent, 200 patients of ages 20-40 years, categorized as American Society of Anesthesiologists I (ASA-I) (normal healthy patients) and ASA-II (mild systemic disease with no functional limitation) undergoing laparoscopic cholecystectomy were enrolled in the study. Exclusion criteria included patients with concomitant co-morbid conditions like diabetes mellitus, hypertension, malignancy, pulmonary, hepatic or renal diseases, patients with $\mathrm{BMI}>35$, patients already taking Pregabalin, gabapenetene, opioid analgesic, long term NSAIDS/COX-2 inhibitors or other pain killers, or those patients in whom use of Pregabalin, Celecoxib or NSAIDS (due allergic/hypersensitivity reactions), or general anesthesia is contraindicated.

All patients were assessed a day before surgery (at least 8 hours before surgery as preoperative anesthesia fitness procedure). After overnight fasting, patients were randomly divided into group $A$ and $B$ by consecutive non- 
probability lottery method. Group A received $100 \mathrm{mg}$ oral Pregabalin 1 hour before surgery and Group B were not given Pregabalin and were regarded as controls. Laproscopic cholecystectomy was performed according to the standard protocol. At the end of surgery, patients were extubated and shifted to the post-anesthesia care unit (PACU) for 24 hours monitoring, where the control of post-operative pain was strictly monitored. The pain score was measured at 4 hours postoperatively by the trainee researcher.

The post-operative pain was assessed by using Visual Analog scale (VAS) graded no pain $(0 \mathrm{~cm} / 0 \mathrm{~mm})$ to worst unbearable pain $(10 \mathrm{~cm} / 100 \mathrm{~mm})$ (Figure1). Patients complaining of moderate to severe pain (pain score 5 or above) in either group at 4 hours or even before postoperatively were given rescue analgesic (Ketorolac $30 \mathrm{mg}$ slow intravenous injection), and the pain score was measured at that point. It was considered end point for that patient regardless of the fact that 4 hours have been passed and whatever the point score was present at that time. SPSS version 17 was used to analyze the data. Continuous variables like age, weight, height, BMI, duration of surgery and pain score at 4 hours were entered as mean $\pm S D$. Categorical variables like gender, pain score and pain category were analyzed as number and percentage. To compare the means, independent ttest was applied. Chi-square and Fischer exact test were used to determine the association between categorical variables. $P$ value $\leq 0.05$ was considered as statistically significant.

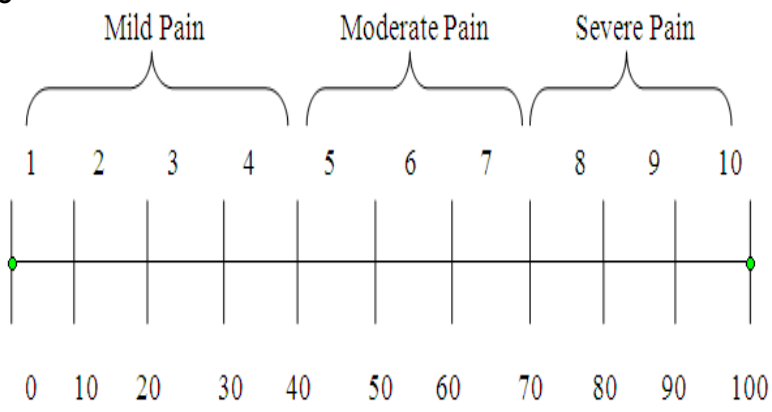

Figure 1: Visual Analog scale for rating of pain intensity

\section{Results}

A total of 200 patients were included in the study. There were 100 patients in each group. The baseline parameters of all study participants are given in Table I.
Overall 198 (99\%) patients complained of mild pain at 4hrs post operatively whereas only $02(1 \%)$ patients had moderate pain. There were no patients with severe postoperative pain at 4 hours.

The baseline comparison of the two groups is given in Table II. Base line characteristics were same between two groups. The mean pain score was significantly high in group B at 4 hrs post operatively. In group B, large number of patients had VAS score of 3 and 4 as compared to group $A$ in which majority of patients had VAS score of 0 , 1 and 2. Regarding VAS pain category, in group $B$ there was no patient with absence of post-operative pain while in group $A$, no patients presented with moderate or severe pain. Frequency of mild pain was approximately equal in both groups (Table III).

\begin{tabular}{|c|c|c|}
\hline \multicolumn{3}{|c|}{ Table I: baseline parameters of all study participants $(n=200)$} \\
\hline \multicolumn{2}{|c|}{ Parameter } & Mean \pm SD \\
\hline \multicolumn{2}{|l|}{ Age (years) } & $33.12 \pm 4.095$ \\
\hline \multicolumn{2}{|l|}{ Weight $(\mathrm{kg})$} & $57.82 \pm 10.865$ \\
\hline \multicolumn{2}{|l|}{ Height $(\mathrm{m})$} & $152.85 \pm 10.293$ \\
\hline \multicolumn{2}{|l|}{$\mathrm{BMI}\left(\mathrm{kg} / \mathrm{m}^{2}\right)$} & $24.67 \pm 2.58$ \\
\hline \multicolumn{2}{|c|}{ Duration of Surgery (hours) } & $48.44 \pm 5.47$ \\
\hline \multirow{2}{*}{\multicolumn{2}{|c|}{ Pain Score at 4 hours }} & $2.48 \pm 1.32$ \\
\hline & & No. of patients (Percentage) \\
\hline \multirow[t]{2}{*}{ Gender } & Male & $38(19)$ \\
\hline & Female & $162(81)$ \\
\hline \multirow[t]{6}{*}{ Pain Score } & 0 & $09(4.5)$ \\
\hline & 1 & $55(27.5)$ \\
\hline & 2 & $32(16.0)$ \\
\hline & 3 & $40(20.0)$ \\
\hline & 4 & $62(31.0)$ \\
\hline & 5 & $02(1.0)$ \\
\hline \multirow[t]{3}{*}{$\begin{array}{l}\text { Pain } \\
\text { Category }\end{array}$} & No & $09(4.5)$ \\
\hline & Mild & $189(94.5)$ \\
\hline & Moderate & $02(1.0)$ \\
\hline
\end{tabular}

Table II: Baseline comparison of the two groups $(n=200)$

\begin{tabular}{|c|c|c|c|}
\hline Gender n (\%) & Group A & Group B & $P$ value \\
\hline Males & $23(60.5)$ & $15(39.5)$ & \multirow[t]{2}{*}{0.15} \\
\hline Females & $77(47.5)$ & $85(42.5)$ & \\
\hline & Mean \pm SD & Mean \pm SD & \multirow[t]{2}{*}{0.52} \\
\hline Age (years) & $32.93 \pm 4.38$ & $33.30 \pm 3.80$ & \\
\hline Weight $(\mathrm{kg})$ & $56.93 \pm 11.53$ & $58.72 \pm 10.14$ & 0.25 \\
\hline Height (m) & $1.53 \pm 0.11$ & $1.52 \pm 0.09$ & 0.80 \\
\hline $\mathrm{BMI}(\mathrm{kg} / \mathrm{m} 2)$ & $24.32 \pm 3.25$ & $25.03 \pm 1.62$ & 0.52 \\
\hline $\begin{array}{l}\text { Duration of } \\
\text { Surgery (min) }\end{array}$ & $48.53 \pm 6.59$ & $48.35 \pm 4.09$ & 0.82 \\
\hline $\begin{array}{l}\text { Pain Score at } \\
\text { 4hrs }\end{array}$ & $1.35 \pm 0.77$ & $3.62 \pm 0.58$ & 0.0001 \\
\hline
\end{tabular}




\begin{tabular}{|c|c|c|}
\hline \multicolumn{3}{|c|}{ Table III: Comparison of different Visual Analog Scores (n=200) } \\
\hline $\begin{array}{c}\text { Visual Analog Scale Pain } \\
\text { Category }\end{array}$ & $\begin{array}{c}\text { Group A } \\
\mathbf{n}(\%)\end{array}$ & $\begin{array}{c}\text { Group B } \\
\mathbf{n}(\%)\end{array}$ \\
\hline 0 & $9(100)$ & 0 \\
\hline 1 & $55(100)$ & 0 \\
\hline 2 & $29(90.6)$ & $03(9.4)$ \\
\hline 3 & $06(15)$ & $34(85)$ \\
\hline 4 & $01(1.6)$ & $61(98.4)$ \\
\hline 5 & 0 & $02(100)$ \\
\hline \multicolumn{2}{|c|}{ Chi square: $p=0.0001$} \\
\hline Visual Analog Scale Pain & $\begin{array}{c}\text { Group A } \\
\text { Category }\end{array}$ & $\begin{array}{c}\text { Group B } \\
\mathbf{n}(\%)\end{array}$ \\
\hline No pain & $9(100 \%)$ & 0 \\
\hline Mild & $91(48.1 \%)$ & $98(51.9 \%)$ \\
\hline Moderate & 0 & $02(100 \%)$ \\
\hline Severe & 0 & 0 \\
\hline \multicolumn{2}{|c|}{ Chi square $p$ value 0.004} \\
\hline
\end{tabular}

\section{Discussion}

Pain is thought to be inadequately treated in one-half of all surgical procedures. Early postoperative pain is the most common complaint after elective laparoscopic cholecystectomy. ${ }^{18}$ Our study participants experienced post-operative pain at $4 \mathrm{hrs}$ after laparoscopic cholecystectomy in more than $90 \%$ cases. The corresponding pain categories were mild in $9(4.5 \%)$ and moderate in 189 (94.5\%) patients. No patients reported severe post-operative pain. Pain is the main reason for overnight hospital stay up to $40 \%$ patients after day care surgery. Postoperative pain is the main reason for delayed recovery after laparoscopic cholecystectomy and major factor for development of "post-laparoscopic cholecystectomy syndrome". 19

In recent years, pregabalin has been introduced as an adjunct in the multimodal management of postoperative analgesia. ${ }^{20}$ According to a meta-analysis, the analgesic effect of pregabalin is best experienced in early postoperative period. ${ }^{21}$ Numerous studies have evaluated the efficacy and adverse effects of pregabalin in reducing acute postoperative pain.21, 22. In our study, Pregablin was found to be an effective drug for post-operative analgesia with 9 (9\%) patients experiencing no pain and 91 (91\%) had mild pain in the pregabalin group. Our findings are congruent with Agarwal and co-workers who also proved that single pre-operative dose of Pregabalin can effectively control the post-operative pain among patients undergoing laparoscopic cholecystectomy. ${ }^{8}$

Evidence also shows that other surgical procedures where pregabalin was administered preoperatively had a clear reduction in post-operative pain. In a recently published article, it was seen that pregabalin use at least one hour prior to Septoplasty reduced post-operative pain significantly, although the required dosage and associated adverse effects need further scientific probing. ${ }^{23}$ According to a set of recent guidelines published by The American Pain society and based on an extensive review of literature, it was suggested that optimal postoperative pain management begins in the preoperative period. This observation is made on the basis of an assessment of the patient and development of a plan of care tailored to the individual and the surgical procedure involved, with follow-up assessments and adjustments as needed. ${ }^{24}$

\section{Conclusion}

Oral pregabalin administered preoperatively, was effective in reducing postoperative pain in patients undergoing laparoscopic cholecystectomy. It can also be concluded that the journey towards good pain control after surgery starts before the actual procedure is initiated. Further studies are needed for post-operative evaluation of side effects, different dosing schedules at different time intervals for both rest and dynamic pain.

\section{References}

1. Vadivelu N, Mitra S, Narayan D. Recent Advances in Postoperative Pain Management. Yale J Biol Med. 2010; 83(1): 11-25.

2. Liu SS, Wu CL. Effect of Postoperative analgesia on major postoperative complications: a systematic update of the evidence. Anesth Analg. 2007; 104(3):689-702.

3. Brennan F, Carr DB, Cousins M. Pain management: a fundamental human right. Anesth Analg. 2007; 105(1): 205-21.

4. Lynch ME, Schopflocher D, Taenzer P, Sinclair C. Research funding for pain in Canada. Pain Res Manag. 2009; 14(2): 113-5. 
5. Bellows C F, Berger D H. Infiltration of suture site with local anesthesia for management of pain following laparoscopic ventral hernia repairs: a prospective randomized trial. JSLS. 2006; 10(3):345-50.

6. Baidya DK, Agarwal A, Khanna P, Arora MK. Pregabalin in acute and chronic pain. J Anaesthesiol Clin Pharmacol. 2011; 27(3):307-14.

7. Peng PW, Li C, Farcas E, Haley A, Wong W, Bender $J$, Chung F. Use of low-dose pregabalin in patients undergoing laparoscopic cholecystectomy. $\mathrm{Br} J$ Anaesth. 2010; 105(2):155-61.

8. Agarwal A, Gautam S, Gupta D, Agarwal S, Singh $\mathrm{PK}$, Singh U. Evaluation of a single preoperative dose of pregabalin for attenuation of postoperative pain after laparoscopic cholecystectomy. $\mathrm{Br} \mathrm{J}$ Anaesth. 2008; 101(5):700-4.

9. Ghafouri A, Movafegh A, Hakimian M, Mehrkhani F, meysamie A. Effect of incisional site infiltration of bupivacaine on post-operative pain and meperidine consumption after midline laparotomy. Iran J Med Sci. 2009; 34(1): 65-7.

10. Cantore F, Boni L, Di Giuseppe M, Rovera F, Dionigi $G$ et al. Pre-incision local infiltration with levobupivacaine reduces pain and analgesic consumption after laparoscopic cholecystectomy: a new device for day-case procedure. Int J Surg. 2008; 6(1): S89-92.

11. Kissin I. Preemptive analgesia. Anesthesiology: The Journal of the American Society of Anesthesiologists. 2000; 93(4):1138-43.

12. Dionne R. Preemptive vs preventive analgesia: which approach improves clinical outcomes? Compendium of continuing education in dentistry (Jamesburg, $\mathrm{NJ}$ : 1995). 2000; 21(1):48-51.

13. Katz J, McCartney CJ: Current status of preemptive analgesia. Curr Opin Anaesthesiol, 2002, 15, 435441.

14. Pogatzki-Zahn EM, Zahn PK. From preemptive to preventive analgesia. Current Opinion in Anesthesiology. 2006; 19(5):551-5.

15. Zhang J, Ho KY, Wang Y. Efficacy of pregabalin in acute postoperative pain: a meta-analysis. $\mathrm{Br} \mathrm{J}$ Anaesth. 2011; 106(4):454-62.
16. Jokela R, Ahonen J, Tallgren M, Haanpää M, Korttila K. A randomized controlled trial of perioperative administration of pregabalin for pain after laparoscopic hysterectomy. Pain. 2008; 134(1-2):10612.

17. Hill CM, Balkenohl M, Thomas DW et al. Pregabalin in patients with postoperative dental pain. Eur J Pain. 2001; 5(2):119-24.

18. Tufano R, Puntillo F, Draisci G, Pasetto A, Pietropaoli $P$, Pinto $G$, et al. Observational Study of the management of mild-to-moderate Post-Operative Pain(ITOSPOP). Minerva Anestesiol. 2012; 78(1):1525.

19. Pergolizzi JV Jr, Raffa RB, Tallarida R, Taylor R, Labhsetwar SA. Continuous multimechanistic postoperative analgesia: a rationale for transitioning from intravenous acetaminophen and opioids to oral formulations. Pain Pract._2012; 12(2):159-73.

20. Şen M, Özol D, Bozer M. Influence of preemptive analgesia on pulmonary function and complications for laparoscopic cholecystectomy. Dig Dis Sci. 2009; 54(12):2742-7.

21. Zhang J, Ho KY, Wang Y. Efficacy of pregabalin in acute postoperative pain: a meta-analysis. $\mathrm{Br} J$ Anaesth. 2011; 106:454-62.

22. Page RL 2nd, Cantu M, Lindenfeld J, Hergott LJ, Lowes BD. Possible heart failure exacerbation associated with pregabalin: case discussion and literature review. J Cardiovasc Med (Hagerstown). 2008; 9(9):922-5

23. Chen N, Soneru C, Kacker A. Does a single dose of pregabalin help with postoperative pain after septoplasty? The Laryngoscope. 2018; 128(5):10234.

24. Chou R, Gordon DB, de Leon-Casasola OA, Rosenberg, J.M., Bickler S, Brennan, T et al. Management of Postoperative Pain: a clinical practice guideline from the American pain society, the American Society of Regional Anesthesia and Pain Medicine, and the American Society of Anesthesiologists' committee on regional anesthesia, executive committee, and administrative council. J Pain. 2016; 17(2):131-57. 